\title{
Fusion of Thermal and Visible Acquisitions for Evaluating Production-borne Scratches and Shunts in Photo-Voltaic PV Cells
}

\author{
Muneeb Tariq ${ }^{1}$, Mahmoud Abdelhamid ${ }^{1}$, Yuntao Li $^{1}$, Mohammed Omar ${ }^{1,2} \&$ Yi Zhou ${ }^{3}$ \\ ${ }^{1}$ Clemson University International Center for Automotive Research CU-ICAR, Automotive Engineering \\ Department, Greenville SC, USA \\ ${ }^{2}$ Masdar Institute for Science and Technology, Masdar City, Abu Dhabi-UAE \\ ${ }^{3}$ Cymer, San Diego, CA, USA \\ Correspondence: M. Omar, Clemson University International Center for Automotive Research CU-ICAR, \\ Automotive Engineering Department, Greenville SC 29607, USA. Tel: 1-864-283-7226, 1-971-2810-9438. \\ E-mail:momar@clemson.edu,momar@masdar.ac.ae
}

Received: July 9, 2012 Accepted: August 13, 2012 Online Published: September 3, 2012

doi:10.5539/jmsr.v1n4p57 URL: http://dx.doi.org/10.5539/jmsr.v1n4p57

\begin{abstract}
This manuscript discusses the development of a fusion-based method of visible and thermal acquisitions (Long Wave and Mid-Wave Infrared LWIR, MWIR) to evaluate scratches and shunts in poly-crystalline, Photo-Voltaic PV solar cells. The proposed technique enables non-destructive and real-time inspection of PV cells production-borne defects; using forward bias and reverse bias Electro-Luminescence EL modes. The thermal imagery of the cells is designed to expose the extent of scratches in both the LWIR and MWIR spectra and whether it acts as an emitter layer bringing the p-n junction to the cell surface; while the visible images (from a CCD detector) evaluate the scratch effect on the surface coating layer and hence its reflectivity. On the other hand, the shunts are detected as hot spots and classified as Ohmic and Non-Ohmic using the reverse and forward bias images of the MWIR imager. The acquired images coming from the three channels (LWIR, MWIR, Visible) are combined using two fusion algorithms suited for real-time applications $(>30 \mathrm{~Hz})$, specifically; Wavelet based fusion, and Principle Component Analysis PCA based fusion. The fused results are further judged based on several reported criteria mainly; cross entropy, mutual information, and the Signal to Noise Ratio SNR, highlighting the need for a unified fusion evaluation approach. The fusion of visible and thermal inputs provides accurate assessment of the PV cells scratches and shunts in contact-less and real-time fashion. The proposed fused imagery approach combines the information from visual inspection and thermal testing using spatially resolved scanning of the PV cell in real-time.
\end{abstract}

Keywords: fusion, principle component analysis, wavelet, photovoltaic cell

\section{Nomenclature}

MWIR Mid Wave Infrared

LWIR Long Wave Infrared

NIR Near Infrared

PV Photovoltaic

LIT Lock-in Thermography

DLIT Dark Lock-in Thermography

CDI Carrier Density Imaging

EL Electroluminescence

PL Photoluminescence

eV Electron Volt

$\mathrm{I}_{\mathrm{F}} \quad$ Fused Image

$\mathrm{I}_{\mathrm{V}} \quad$ Visible Image 


$\begin{array}{ll}\mathrm{I}_{\mathrm{M}} & \text { MWIR Image } \\ \mathrm{I}_{\mathrm{L}} & \text { LWIR Image } \\ \text { SNR } & \text { Signal to Noise Ratio } \\ \lambda_{\max } & \text { Peak wavelength } \\ \mathrm{T} & \text { Temperature } \\ \mathrm{M}_{\mathrm{e}, \lambda} & \text { Spectral Exitance } \\ \mu_{\mathrm{n}} & \text { Mean of Image } \\ \sigma_{\mathrm{n}} & \text { Standard Deviation of Image } \\ \mathrm{CE} & \text { Cross Entropy } \\ \text { SF } & \text { Spatial Frequency } \\ \text { PSNR } & \text { Peak Signal to Noise Ratio }\end{array}$

\section{Introduction}

Crystalline silicon Photovoltaic (PV) cells are the oldest PV technology, which still dominates the current PV industry (Galland, 2012) and (NREL, 2012). These cells are subdivided into mono and polycrystalline silicon PV cells. Crystalline PV cells are either grown as single crystals using the "Czochralski Cz" process and the "Float-Zone" technique FZ or casted into ingots, which are then mechanically cut. While such cells may also be excised from multifaceted silicon crystals, this induces non-uniformity in their appearance and degrades performance. Poly (Multi) crystalline silicon ( $\mathrm{m}-\mathrm{Si}$ ) wafers fabrication starts by a wire sawing or cutting a block-cast silicon ingots into thin $\sim 180-300 \mu \mathrm{m}$ slices. Then n-type dopants are diffused upon the front (light-receiving) side of the slice, to build a p-n junction a few hundred nanometers below the surface. A coating process applies a thin layer $\sim 100 \mathrm{~nm}$ of an anti-reflective paint (typically Silicon-Nitride) to increase the light penetration into the active cell surface while preventing any recombination losses. The coating is typically done through an Enhanced Chemical Vapor Deposition PECVD process. The wafer then has a full area metal contact made on the back surface. A grid-like metal contact comprised of fine "fingers" and larger "bus-bars" are screen-printed onto the front surface using a silver paste, and aluminum paste is screen-printed onto the rear contact (Saga, 2010) and (Ranjan, Balaji, Panella, \& Ydstie, 2011).

The performance of silicon PV cells is greatly curbed by recombination via defects and impurities in the bulk. This holds in particular for solar cells produced on multi-crystalline silicon (mc-Si) wafers, containing large concentrations of crystallographic defects, such as dislocations and grain boundaries (Tachibana et al., 2012), as well as recombination-active impurities, such as transition metals. Also hampering the PV cell total efficiency (the product of its thermodynamic, reflectance, charge carrier, and conductive efficiencies (Saga, 2010)) are the induced defects of the fabrication-process. This imperfect process, which involves the fabrication of mono (c-Si) and multicrystalline Silicon (m-Si) cells from larger silicon ingots via mechanical cutting, results in micro-cracking (St-Laurent, Genest, Simon, \& Maldague, 2004), with the small size of these cracks making visual detection impractical, especially at high production cadences. Furthermore, parasitic shunt resistances, emanating from manufacturing defects that form at the wafer edges between the active layers, are an inherent problem in PV cell production (Stay, 2007). Such low resistance shunts, which reduce the Fill Factor FF and Open Circuit Voltage $\mathrm{V}_{\text {oc }}$ of the cell, cause power losses by providing an alternate current path for the light generated current. These shunts may be induced at various points in the manufacturing process (e.g. poor initial silicon quality, contact over firing, trace metal deposition at the cell edge, etc.). While several conventional technologies (e.g. laser cutting, diamondsaw or plasma etching) are useful in preventing shuntresistance, they may not provide adequate results, either due to excessive heat damage (as with conventional lasers) reduction in the fracture strength of thewafer from sawing, or from the high-priced etching procedures (Stay, 2007). Other process-induced shunts include nonlinear edge shunts, which are interpreted as recombination sites; and Schottky-type shunts, which result from sintered metallization causing direct contact between the metal and p-type base material (Acciani, Falcone, \& Vergura, 2010) and (Breitenstein, Rakotoniaina, Al Rifai, \& Werner, 2004).

In this study, the focus will be on scratches at the surface of a PV cell across an emitter layer, which brings the $\mathrm{p}$-n junction to the surface with a high density of recombination sites, thusly mimicking a nonlinear edge shunt (Acciani et al., 2010). In addition reverse and forward bias currents will be used to detect and categorize Ohmic and Non-Ohmic PV shunts. The structure of this manuscript discusses the different inspection routines currently used for PV cells while being manufactured, in section two. Section three addresses the experimental setup, 
while section four introduces the proposed imagery fusion methodology. The result and discussion is in sections five and six; lastly, the study findings are presented and summarized in the conclusion section.

\section{Current Inspection Techniques}

Thermography has been widely used to test PV cells for defect detection but the sensitivity and thermal resolution of standard thermography, however, is limited by the inherent detector sensitivity (Noise Equivalent Temperature Difference). Conventional Infrared imaging techniques use heating or an excitation source to inspect PV cells, resulting in significant thermal diffusivity that negatively affects spatial resolution. To minimize this thermal diffusion pulsed or sinusoidal stimulation is used which can be in the form of light or electric signals. Lock-in thermography (LIT) (Bauer, Breitenstein, \& Wagner, 2009) is a technique that uses a modulated excitation source which is synchronized to the data acquisition of the camera for obtaining a sequence of images. Thisallows the system to detect subtle thermal responses beyond the noise floor limitations of the infrared camera. Furthermore, Lock-in thermography allows mapping of forward current density distribution and is also useful in revealing series resistances and sites of high carrier recombination. As a result, charge carrier behavior in PV cells can be characterized with Lock-in thermo-graphy systems by using carrier density imaging (CDI); which maps saturation current density over an entire PV cell. Dark Lock-in thermography (DLIT) (Bauer et al., 2009) is a useful technique to detect shunts, which are defects that leak current and arecreated during solar cell processing. These shunts can be detected by examining a PV cell's I-V (current-voltage) characteristics. In DLIT the cell under inspection is put under reverse bias; while the shunts are detected by its thermal signature, which is generated by current leakage across the p-n junction. An ideal solar cell under reverse bias would have very little current flow. However, imperfections in the cell often lead to areas where the cell's junction is at a lower resistance, causing current to flow through it. This flow of current then generates heat, creating hotspots.

Characterization techniques that will quantitatively allow the spatial variation in material quality to be quickly assessed are a valuable tool for in-line process monitoring and production of high efficiency PV cells. Photoluminescence PL imaging with the Short-Wave to Mid-Wave Infrared (SWIR-MWIR) appliance is another widely used nondestructive, contactless testing technique, allowing variations of the minority carrier lifetime within silicon wafers to be measured with high spatial resolution and small data acquisition time. Here, the photovoltaic cells are illuminated under high optical power at one wavelength causing the absorption of photons into the bulk material. This absorption results in some energy loss as heat during the interaction with the molecular structure of the photovoltaic device. The remaining energy then causes the reemission of photons at longer wavelengths, and an infrared detector is used to detect any defects in the resulting image (Malchow, 2009).

Trupke et al. (2007) showed effective minority carrier lifetimes from calibrated PL images of various mono-crystalline PV cells undergoing different processing stages such as; etching, emitter diffusion, anti-reflective coat deposition and fully processed PV cells, to highlight the possibility of monitoring the influence of individual processing steps on local material parameters. Furthermore, PL imaging can be used to fairly accurately identify the position of localized shunts in PV cells which may later be isolated to increase cell performance as described in (Schmidt, Pohl, Bothe, \& Brendel, 2007) and (Augarten et al., 2007). Shunted areas have reduced luminescence intensity in both PL and EL images and are often characterized with blurred regions in vicinity of shunts. This blurring is a result of voltage drops across shunts associated with lateral current flow through the emitter. Moreover, dislocations within the wafer of PV cells also limit the cell efficiency. These structural defects are linked to trap density and ultimately affect the minority carrier lifetime of the cell (Schmidt et al., 2007). Trupke et al. (2007) also showed defect luminescence imaging on string ribbon silicon wafer to identify efficiency limiting dislocation density early on in the cell process. In addition to this, PL imaging is particularly appealing for crack detection due to its non-contact nature and fast data acquisition technique whose application is suitable before and after any processing stage (Trupke, Bardos, Schubert, \& Warta, 2006). Using ideal conditions on sister wafers, i.e. neighboring wafers cut from the same ingot, Trupke et al. (2007) distinguished cracks from superficial scratches or material induced defects by enhancing the sensitivity and reliability of PL imaging for crack detection. Material induced defects such as grain boundaries and dislocations would have similar prominent features in adjacent wafers cut from the same ingot. However, cracks, mainly micro-cracks, are unlikely to have the same shape or be present in adjacent wafers.

On the other hand, Electroluminescence EL is also useful in detecting various anomalous behaviors in a PV system. In electroluminescence, the current flows in a forward bias mode through the PV system which causes minority carriers to recombine and generate photon emissions ( $1.1 \mathrm{eV}$ for silicon). The resulting light from the activity of the minority carriers is then collected and imaged. EL signals are obtainable with integrating Charged Coupled Device CCD or Indium Gallium Arsenide InGaAs type imagers as discussed in (Colvin, 2009). For a 
detailed examination of cell performance, an important material parameter to be monitored is the minority carrier diffusion length, which governs the collection efficiency. Würfel et al. (2009) used EL to give a quantitative determination of the spatially resolved diffusion length of screen printed mc-silicon cell from the ratio of two luminescence images measured using two different spectral filters. Fuyuki, Kondo, Yamazaki, Takahashi and Uraoka (2005) also explained the proportional relation between the emission intensity and minority carrier diffusion length. Even though emission intensity is affected by many physical properties such as surface recombination velocity and the recombination at defects, etc., Fuyuki, Kondo, Yamazaki, Takahashi and Uraoka (2005) considered that emission intensity will be proportional to the number of minority carriers, which is determined by the diffusion length. The shunt detection capabilities of luminescence imaging methods are investigated in Kasemann et al. (2008) by relating the luminescence intensity to the local junction voltage. Localized shunts drain majority currents from its surroundings, which eventually leads to a drop in voltage across series resistances that connect shunts laterally to non shunted areas. Hence, as a result of the voltage drop a 'blurred' intensity dip can be seen in luminescence images. So, the defects accompanying the PV cells production can be inspected using different modalities. Some scratches and facial non-uniformities can be identified using normal visual inspection, while some may require the use of spatially resolved sub-facial measuring techniques, such as thermal testing methods. Image fusion can help combine information from these two different techniques onto a single full-field image for further analysis.

\section{Experimental Setup}

This study utilizes an Electroluminescence EL inspection mode in forward and reverse bias excitations. The setup mainly consists of three camera systems, as shown in Figure 1, a power supply and a mono-crystalline silicon wafer PV cell. The imagers used comprise of a board Charged Coupled Device CCD camera with a spatial resolution of $219 \times 312$ pixels and an acquisition frequency of $30 \mathrm{~Hz}$. The thermal imagers are a photonic Mid-Wave Infrared MWIR camera, commercial name Indigo-Phoenix, product of FLIR, MA. This infrared detector has an Indium Antimonite InSb Focal Plane Arrays FPA with a spectral sensitivity range between 1.5-5.0 microns and a pixel format of 640 x 512 pixels. This MWIR detector's Noise Equivalent Temperature Difference NETD is 0.1 mili-Kelvins at room temperature. Also, a Long-Wave Infrared LWIR imager is used; this detector is based on a micro-bolometric array of $320 \times 240$ pixels with a temperature range up to $500^{\circ} \mathrm{C}$, with an NETD of $0.1 \mathrm{~K}$. Additionally, the three camera setup is mounted on an XYZ mechanism to translatethe scanning across larger PV cell arrays using servo-motors, if needed.

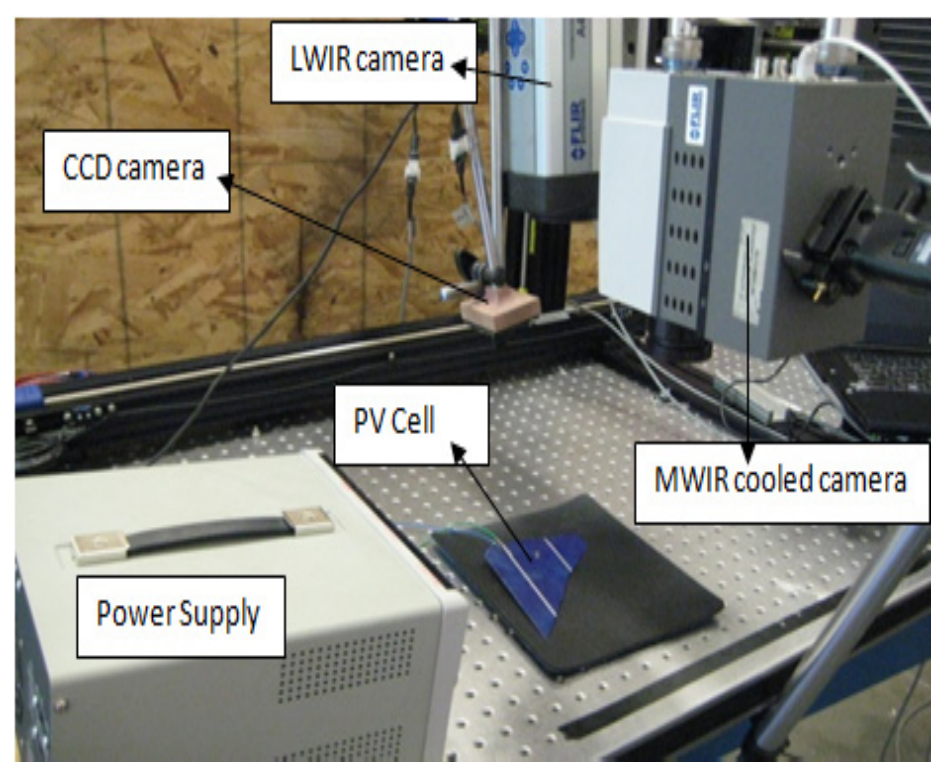

Figure 1(a). Setup of Electroluminescence showing three different channels for imaging 


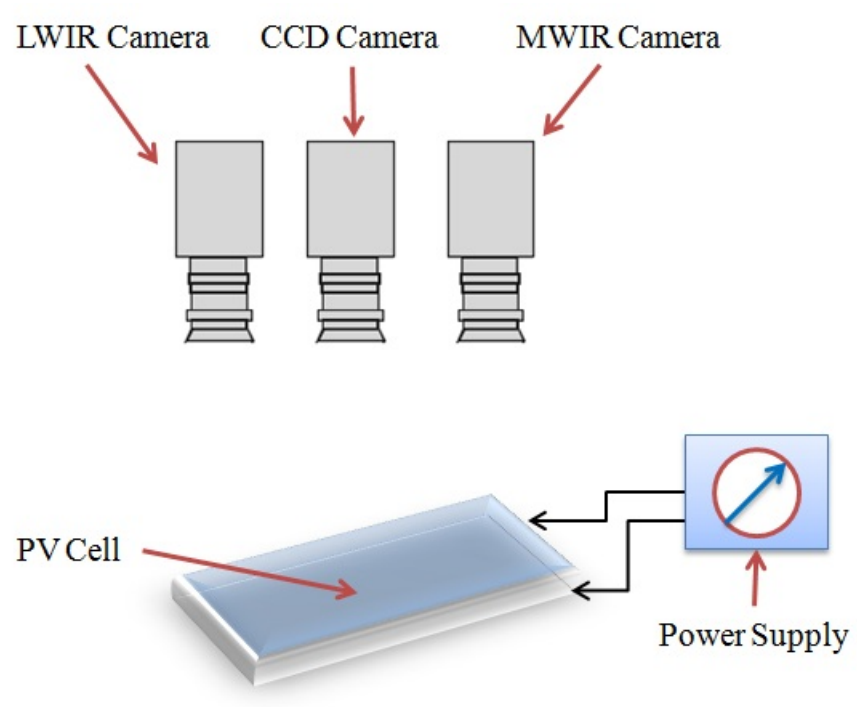

Figure 1(b). Schematic diagram for experimental setup

The Electro-Luminescence EL experiment supplies the mono-crystalline silicon wafer, via their metal contacts, with an external excitation current using a DC power supply, while the detectors acquire images of the cell. The DC power supply triggers $870 \mathrm{~mA}$ of current at an operating voltage of 0.55 Volts to the cell for this particular scenario. The three imaging devices Field of Views FOV are calibrated to ensure coverage of the same scene; in other words, the detectors' registration is done at the hardware level. The CCD camera assists in identifying defects limited to the surface of the PV cell, while, the infrared cameras can identify sub-surface defects. A superficial scratch is fabricated on the PV cell to account for the surface defects. On the other hand, the infrared cameras would assist with highlighting intrinsic defects, such as, localized defects due to impurities, shunts, cracks, etc. The PV cell as seen by the CCD camera with the superficial scratch circled is shown in Figure 2.

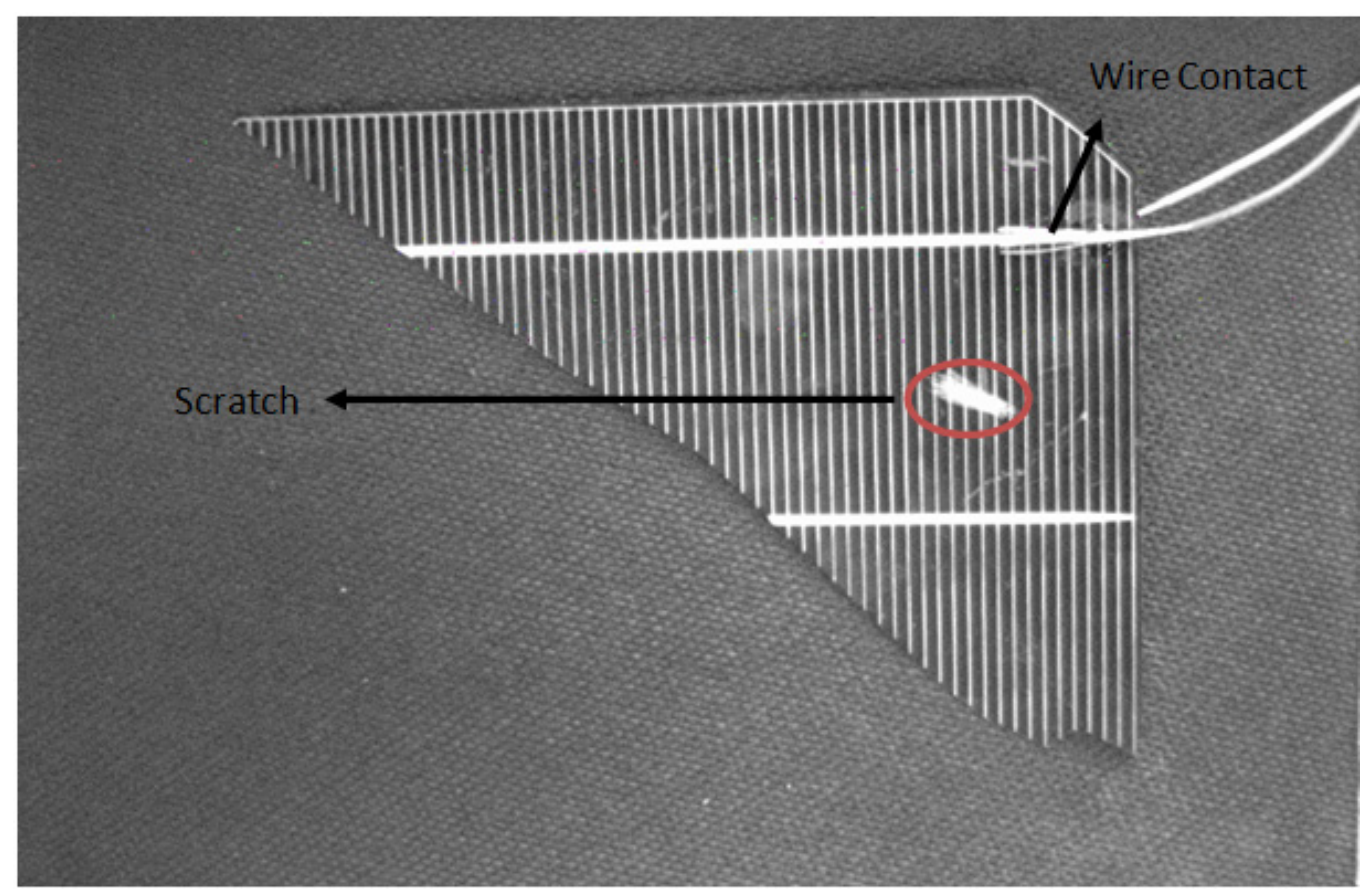

Figure 2. Visual Image of PV cell as seen by the CCD camera 


\section{Introduction and Characterization of Image Fusion}

Image fusion combines the information from the three image sources corresponding to the same scene but from three different spectral views, with the intention to retain the most desirable characteristics from each image. Generally, image fusion can be implemented at three different levels or categories; the pixel level, the feature level, and the decision level as discussed in (Lanir, Maltz, Yatskaer, \& Rotman, 2006). In (Zhou, Mayyas, Qattawi, \& Omar, 2010) described the details of these three levels in addition (Zhou \& Omar, 2009) provide a comprehensive review of the different fusion methodologies. The roposed study will only implement the pixel level fusion due to its advantages for current scenario; pixel level fusion is effective for high quality raw images but not suitable for images with unbalanced quality level because the information from a physical channel might be impeded by the other. In pixel level fusion, the input images are fused pixel by pixel; with the aid of a set of arithmetic operations, or Multi-Scale Decomposition MSD, or false color domain operations. The basic block diagram for a pixel level fusion scheme is displayed in Figure 3.

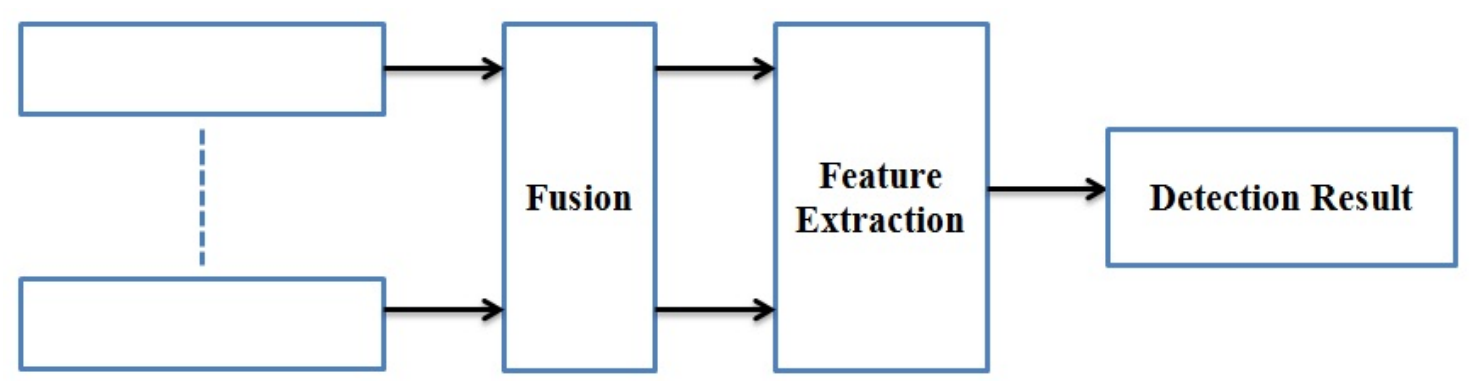

\section{Registered Input Images}

Figure 3. Pixel level fusion scheme

Typical arithmetic operations include: (1) Weighted average, and (2) Comparison. In weighted average the fusion is done based on the weights assigned to each image source. The weights may be fixed or variable based on the specific application, dynamic or static relevance as described in Equation (1).

$$
\begin{gathered}
I_{F}(x, y)=\omega_{M}(x, y) \times I_{M}(x, y)+\omega_{L}(x, y) \times I_{L}(x, y)+\omega_{V}(x, y) \times I_{V}(x, y) \\
\omega_{M}(x, y)+\omega_{L}(x, y)+\omega_{V}(x, y)=1
\end{gathered}
$$

Where, $I(x, y)$ is the pixel intensity at location $(x, y) ; \omega(x, y)$ is the weight at location $(\mathrm{x}, \mathrm{y})$; the subscripts $F, M, L, V$ representFused image, MWIR image, LWIR image, and Visual image.

Adaptive weighted average fusion is a variable weight scheme, where the assigned weights to the image change constantly. An algorithm for a three-source adaptive weighted fusionis in Equation (2);

$$
I_{F}(x, y)=\frac{\alpha \times I_{M}(x, y) \times I_{L}(x, y) \times I_{V}(x, y)}{I_{M}{ }^{2}(x, y)+I_{L}{ }^{2}(x, y)+I_{V}{ }^{2}(x, y)}
$$

Where, $I(x, y)$ is the pixel intensity at location $(x, y)$; the subscripts $M, L, V, F$ represent the MWIR, LWIR, Visual and fused image, respectively. $\alpha$ is a coefficient dependent on the specific fusion scenario.

On the other hand, in comparison based fusion method, the maximum or minimum intensity at each pixel location is selected from the input images for the output image as described in Equation (3). However, the comparison based fusion is not suitable for images with saturated pixels.

$$
\begin{gathered}
I_{F}(x, y)=\operatorname{Max}\left(I_{M}(x, y), I_{L}(x, y), I_{V}(x, y)\right) \\
I_{F}(x, y)=\operatorname{Min}\left(I_{M}(x, y), I_{L}(x, y), I_{V}(x, y)\right)
\end{gathered}
$$

Due to the need for a real-time fusion subroutine, two implementations are developed in this study to accompany the pixel level fusion. The first uses a Principle Component Analysis PCA transformation to help reduce the data content while highlighting itsdata variance and reducing its covariance. The second is based on Wavelet compression. 


\subsection{PCA Fusion}

PCA is a statistical tooltypically used for dimension reduction becauseitprojects the data from its original space to its Eigen space to increase the variance and reduce the covariance so as to identify patterns in data. The author in (Rajic, 2002a, 2002b) proposed using PCA in processing industrial thermograms to enhance the defectives' contrast. Sinha, Balas, Ostrovsky and Russell (2006) developed a PCA based feature-level image fusion in face recognition using both IR and visible images. The flow chart of a typical PCA calculation is shown in Figure 4. However, the implementation in Figure 4 is not practical for real-time applications so a modified Singular Value Decomposition SVD transformation is used to compute the principle components, complete mathematical details are in (Parvataneni, 2009). One can also assign weights to the three input images to still achieve weighted average fusion while using the Eigenvalues generated from the PCA. This implementation can be termed a PCA weighted average fusion (Zhou, 2010).

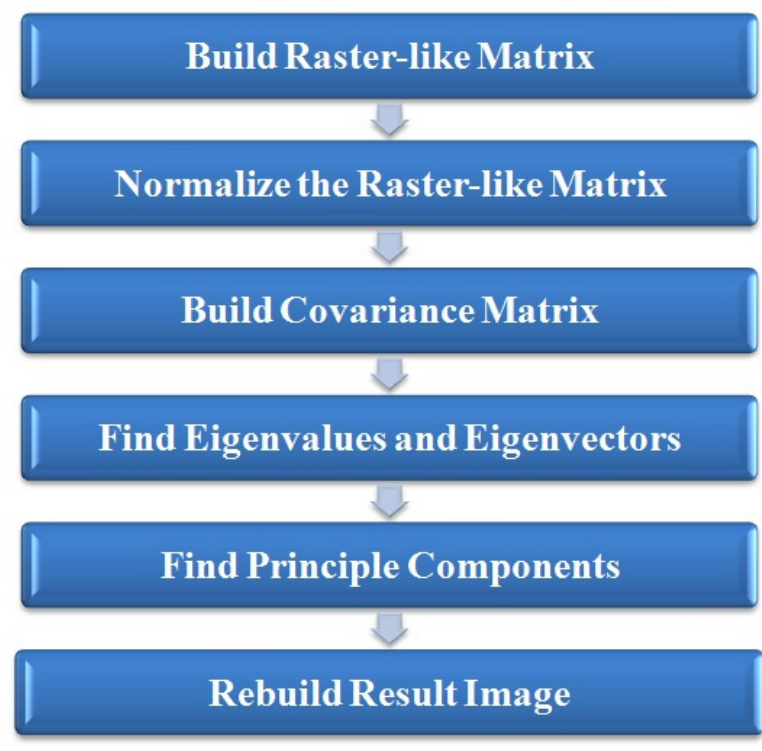

Figure 4. Flow chart of original PCA

In this implementation, the weights for MWIR image, LWIR image, and Visual image are not fixed. Instead, a practical way to decide on the values of $\omega_{M}, \omega_{L}, \omega_{V}$ is to use the eigenvalues of the raster-like matrices of the MWIR image, LWIR image and visible image as described in Equation (4).

$$
\omega_{V}=\frac{E_{V}}{E_{M}+E_{L}+E_{V}}, \omega_{M}=\frac{E_{M}}{E_{M}+E_{L}+E_{V}}, \omega_{L}=\frac{E_{L}}{E_{M}+E_{L}+E_{V}}
$$

Where, $E_{V}$ is the eigenvalue of the visual image, $\mathrm{E}_{\mathrm{M}}$ is the eigenvalue of MWIR image and $E_{L}$ is the eigenvalue of LWIR image. Therefore, this scheme allows combiningthe PCA image contrast enhancementfeature along with the advantages of the weighted average; thusly a new PCA based fusion scheme is developed for the three-channel images as depicted in Figure 5.

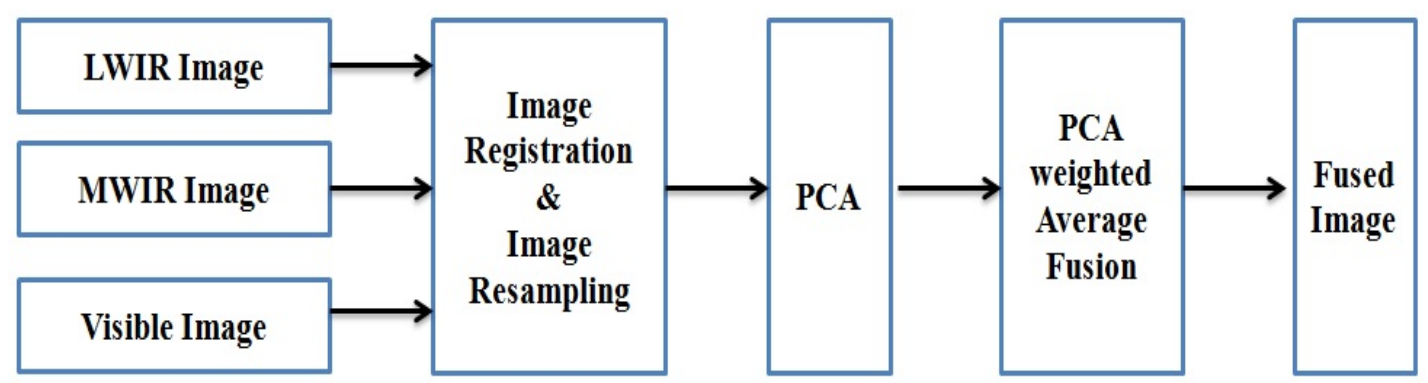

Figure 5. A new PCA based three-channel fusion scheme 


\subsection{Discrete Wavelet Transformation DWT Fusion}

The second possible real-time fusion scheme is the wavelet based fusion, shown in Figure 6 as a block diagram. In DWT based fusion (Pajares \& de la Cruz, 2004), (Hong \& Zhang, 2003), (Hui, Manjunath, \& Mitra, 1994) and (Hill, Bull, \& Canagarajah, 2005), wavelet transforms are applied on the three input images, respectively. In the first level of DWT, each row of the image $I(x, y)$ is horizontally blurred and down sampled by a low pass filter $\mathrm{L}$ and a high pass filter $\mathrm{H}$ while the coefficient matrices, $I_{L}(x, y)$ and $I_{H}(x, y)$ are created. Thenthe DWT vertically blurs and down sampleseach column of $I_{L}(x, y)$ and $\mathrm{I}_{\mathrm{H}}(\mathrm{x}, \mathrm{y})$ to create four coefficient matrices: $I_{L L}(x, y), I_{L H}(x, y), I_{H L}(x, y)$ and $I_{H H}(x, y)$. Afterwards, each column of $I_{L}(x, y)$ and $I_{H}(x, y)$ are vertically blurred and downsampled to create four coefficient matrices: $I_{L L}(x, y), I_{L H}(x, y), I_{H L}(x, y)$ and $I_{H H}(x, y)$. After that, the first level of DWT is accomplished; then the second level of DWT is formed by applying the same process. $I_{L L}(x, y)$ is a blurred and downsampled result of the original image $I(x, y) . I_{L H}(x, y), I_{H L}(x, y)$ and $I_{H H}(x, y)$ are detail sub images representing the horizontal, vertical and diagonal directions of the original image $I(x, y)$. Thus, a DWT with $\mathrm{N}$ decomposition levels will have $M=3 N+1$ such frequency bands. After decomposition, an image fusion decision criterion is generated based on merging the wavelet coefficients. The process of merging the coefficients in an appropriate way in order to obtain the desirable result is the key step of wavelet based image fusion. The simplest way is to take the average of the coefficients to be merged, but there are a lot of other merging strategies. Finally, an inverse wavelet transform is used to generate the fused image. An example of one level and two levels DWT is shown in Figure 7(a) and 7(b) using images from a PV cell.

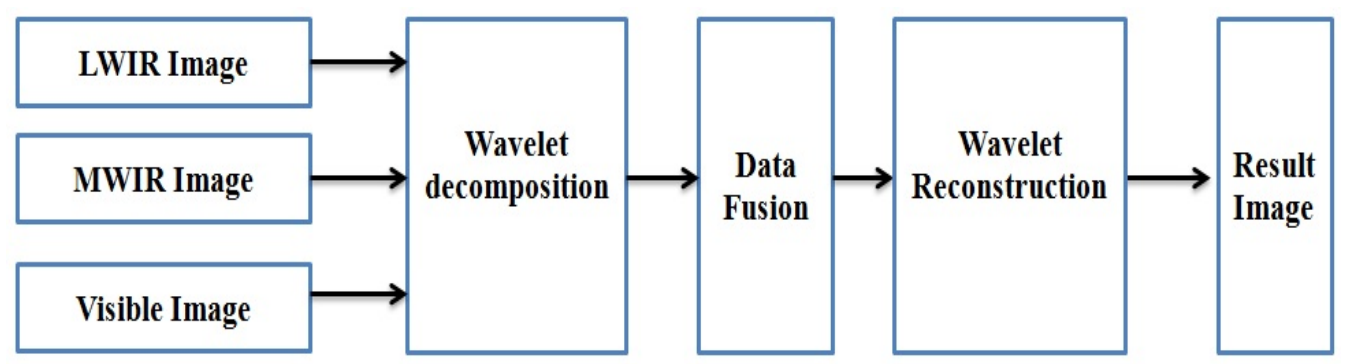

Figure 6. Block diagram of wavelet based fusion scheme for three input images

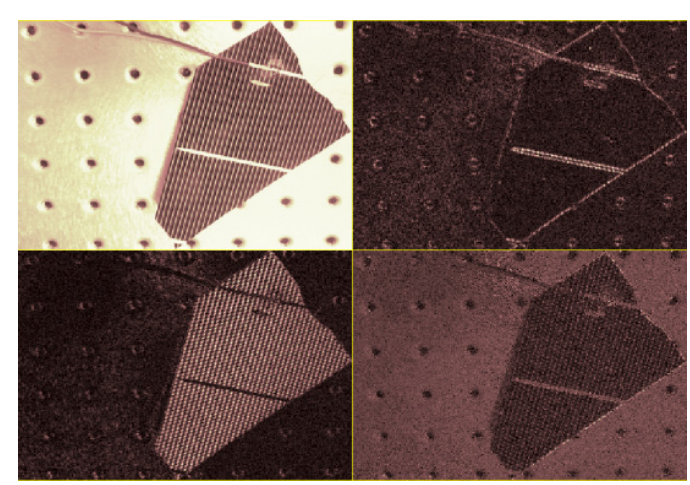

(a)

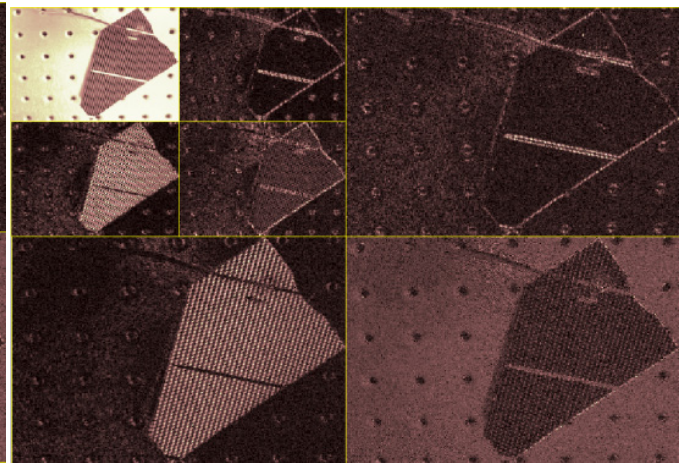

(b)

Figure 7. A representation of (a) one-level and (b) two-level image decomposition

\section{Experimental Data and Results}

Figure 8 shows the thermal image of the PV cell as acquired by the LWIR detector, with the inspected cell being operated at a forward bias current of $870 \mathrm{~mA}$ and a voltage of $0.6 \mathrm{~V}$. A series of thermal images of PV cell using MWIR imager are collected using an integration time of $1.5 \mathrm{~ms}$. The inspected cell operates at a forward bias current of $890 \mathrm{~mA}$ and a voltage of $0.6 \mathrm{~V}$. The thermal image displayed in Figure 9 (a) is taken after 5 sec acquisition time, with the temperature profile shown in degrees centigrade, and the scratch is encircled, while in Figure 9(b) the image is taken after $\mathrm{t}=15$ acquisition period. It can be seen that at different acquisition time the temperature of the PV cell remains uniform in the forward bias mode. 


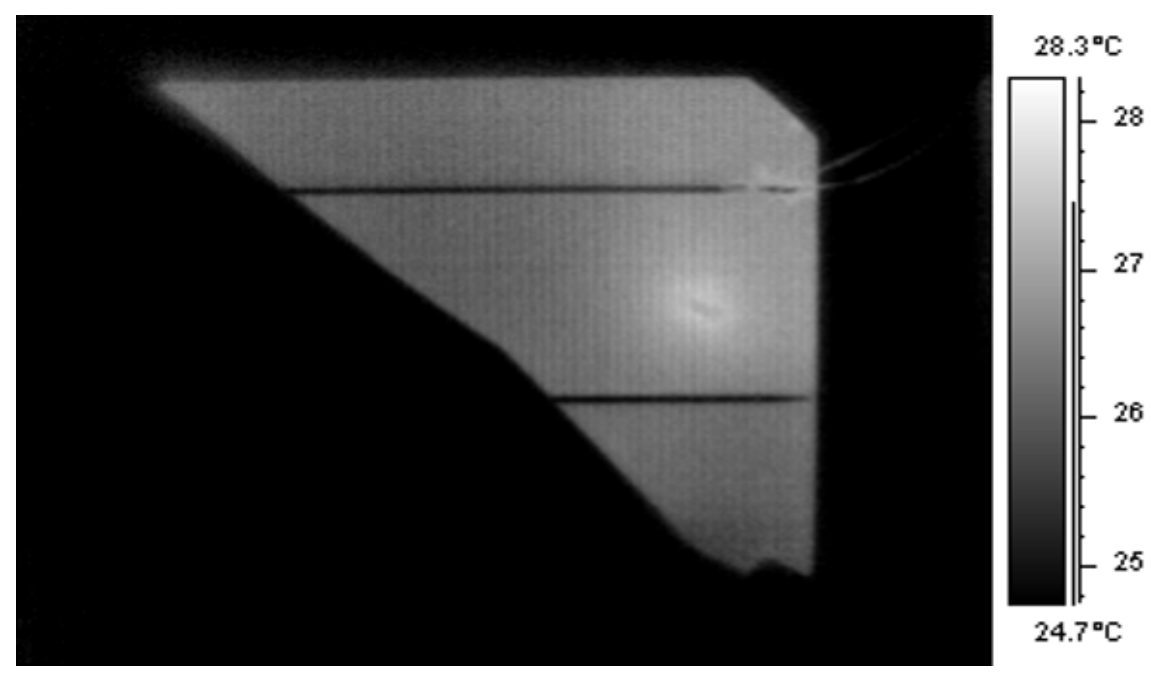

Figure 8. Image of the PV cell as seen by the LWIR camera

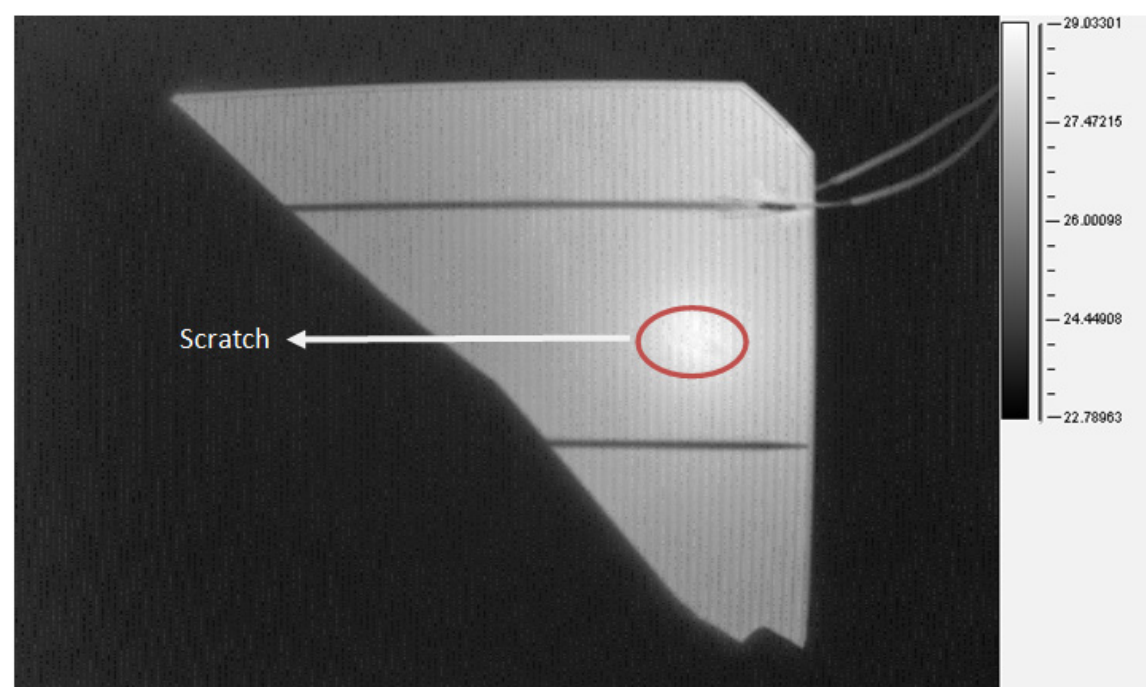

Figure 9(a). MWIR image at $\mathrm{t}=5 \mathrm{sec}$ into acquisition period

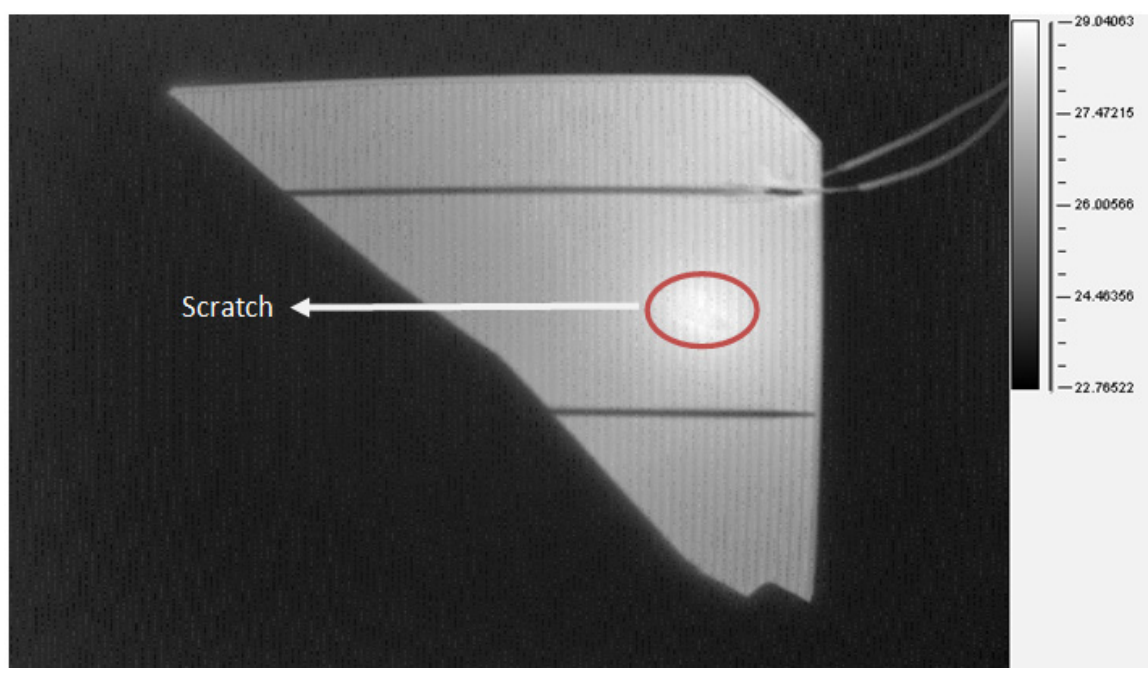

Figure 9(b). MWIR image at $\mathrm{t}=15 \mathrm{sec}$ into acquisition period 
Figure 10(a) shows the thermal image of the PV cell as viewed by the MWIR InSb detector, when the cell is being excited using a reverse bias current of $340 \mathrm{~mA}$ and a voltage of 9.1V. Data acquisition is done over a time period of 20 seconds. This image is taken at $\mathrm{t}=5 \mathrm{sec}$ into the data acquisition period at the same integration time of $1.5 \mathrm{~ms}$. Another image at $\mathrm{t}=15 \mathrm{sec}$ into the data acquisition period is shown in Figure $10(\mathrm{~b})$. Observation shows that the PV cell temperature profile is not constant during the two different acquisition times with significant temperature changes are observed. Furthermore, the PV cell temperature in the vicinity of defects is much higher in reverse bias mode when compared to forward bias mode of operation. To further investigate the shunts (encircled in blue) as shown in Figure 10(a), numerous ROIs (Region of Interest) are investigated to quantify the change in temperature across different shunts. A line profile is generated; as shown in Figure 10(c), across the shunted areas to investigate the change in temperature across the shunts. Figure 11 shows a fused image of the PV cell from three different channels. Image from the visual CCD camera, forward bias LWIR image and reverse bias MWIR image are fused together using a weighted average pixel level fusion. For the image, the "Weight of raw visual image $=0.2$ ", "Weight of LWIR image $=0.2$ " and "Weight of MWIR image $=$ 0.6". The edge shunts along with the superficial scratch is clearly observed. Furthermore, the fused image helps to better locate the superficial scratch and qualitatively shows the thermal spread around it.

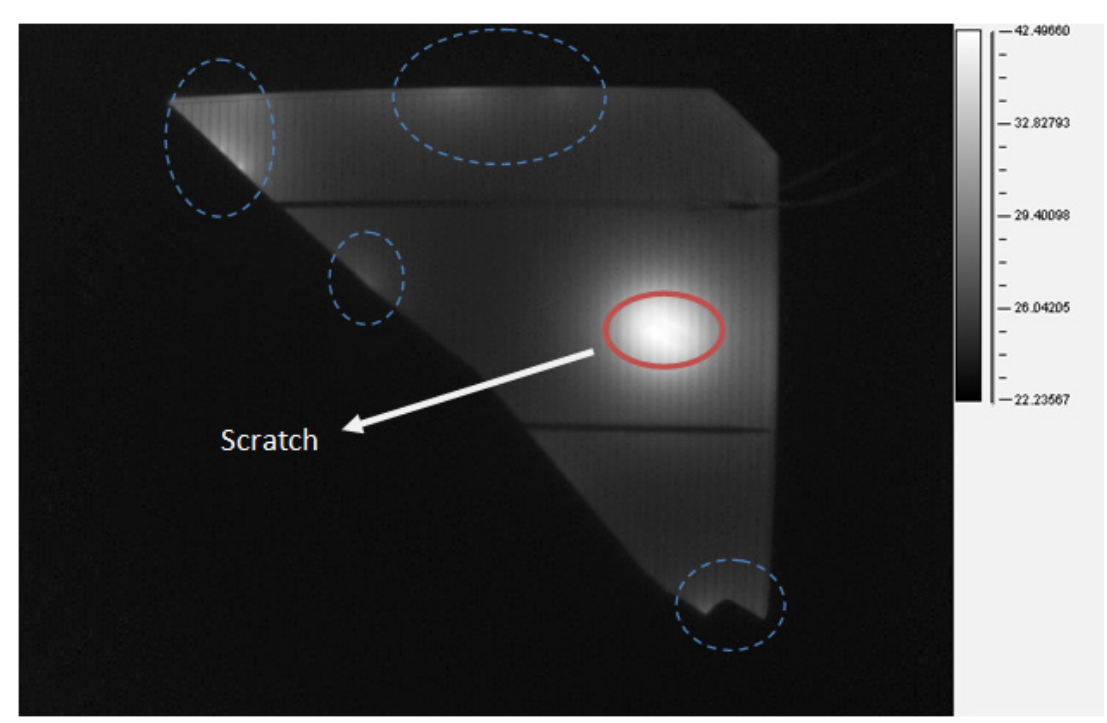

Figure 10(a). Image from MWIR camera under reverse bias conditions at $\mathrm{t}=5 \mathrm{sec}$ into acquisition

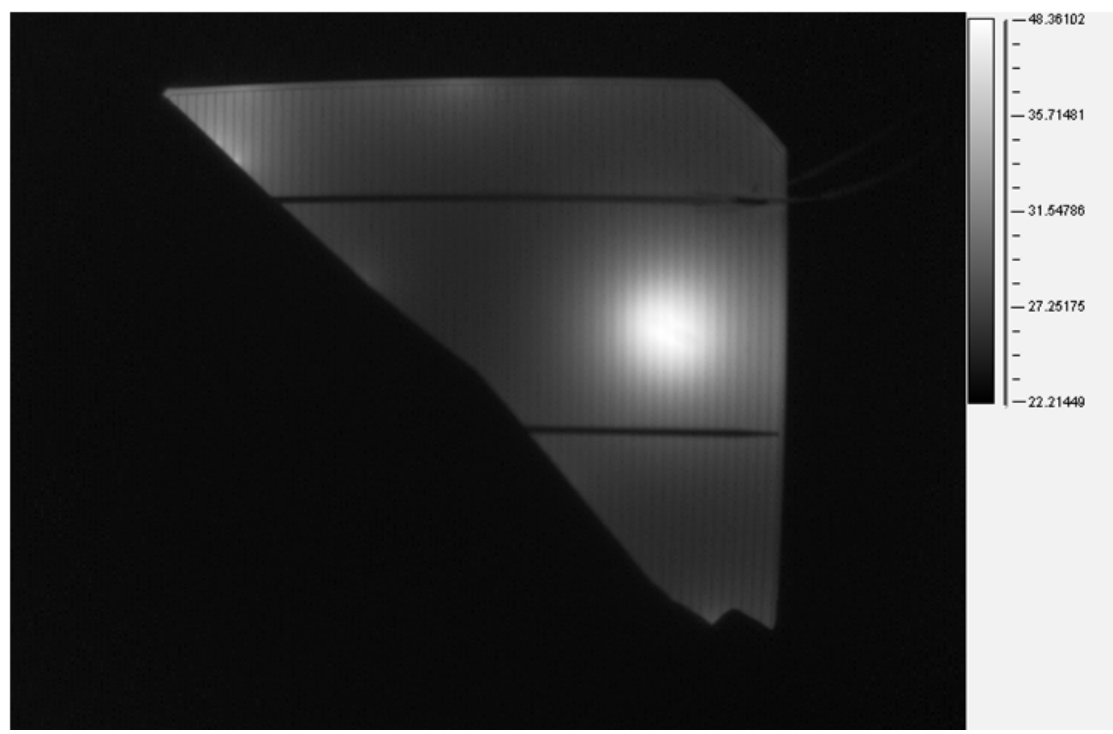

Figure 10(b). Image from MWIR camera at $\mathrm{t}=15 \mathrm{sec}$ into acquisition 


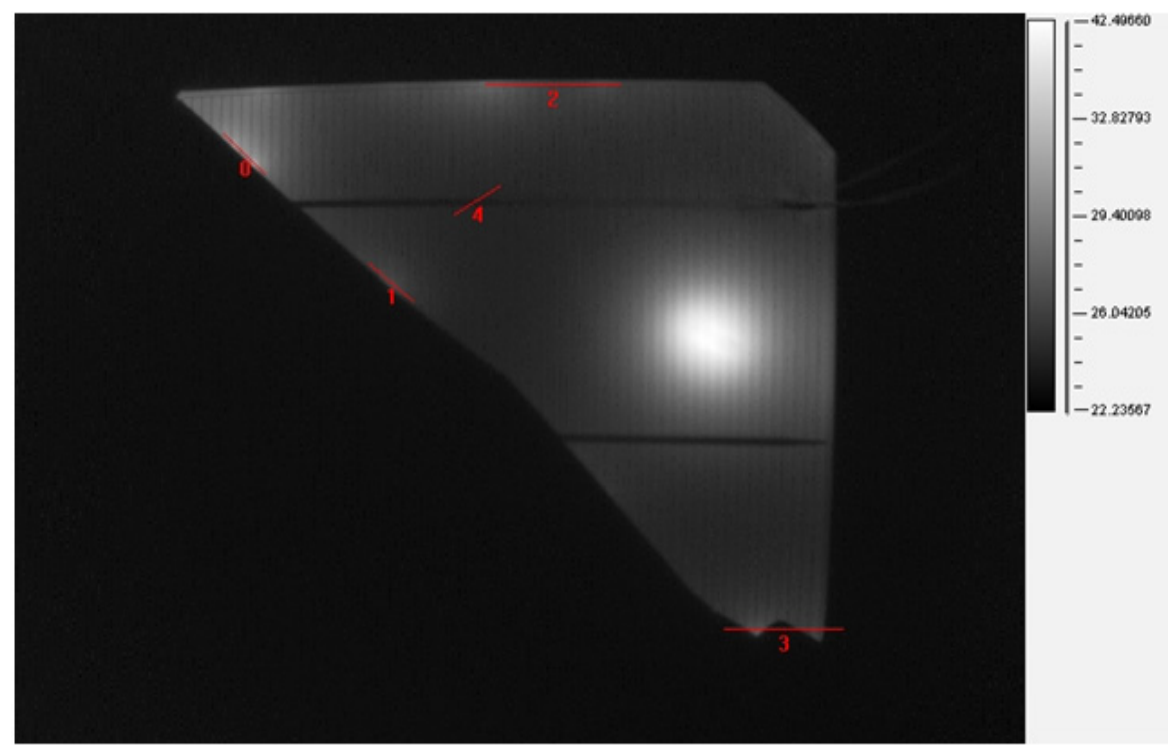

Figure 10(c). MWIR image under reverse bias with various ROIs

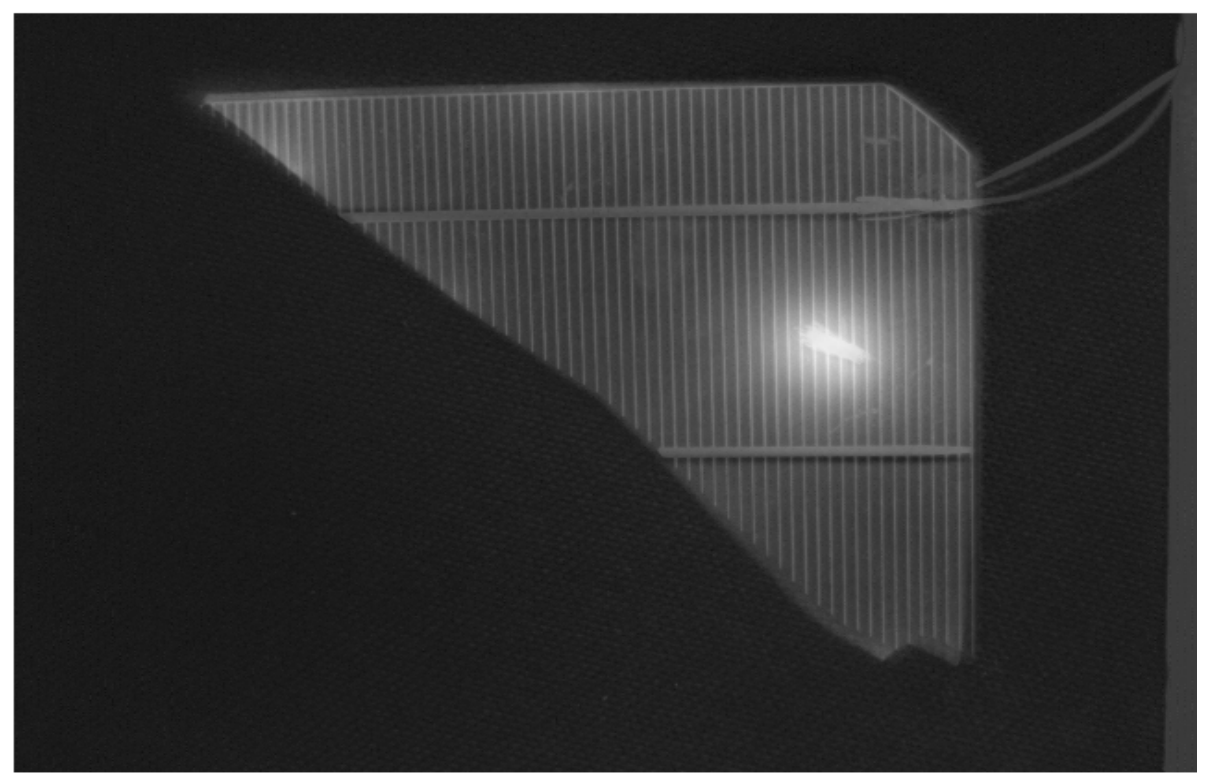

Figure 11. PCA fused image of PV cell from three channels; LWIR, MWIR and CCD camera

\section{Discussion and Analysis}

One of the important PV cell materials attributes is its transmissivity or transmission, which is a measure of throughput of the incident light. The imagesacquired in the MWIR spectra are preferred over those from the LWIR, due to the transmittance of light through silicon which lies well between the 3-5 $\mu \mathrm{m}$ or Mid-Waverange. Figure 12 shows the percentage transmission of light as a function of wavelength for silicon and Figure 13 shows the transmission as effected by temperature. Moreover, this transmittance of light is the highest for silicon near room temperature between the 3-5 $\mu \mathrm{m}$ ranges. An LWIR system would be superior to the MWIR system for a wide span of temperature because of its very broad dynamic range capability. However, the PV cell does not span a very wide temperature change, hence, MWIR is sufficient for yielding better results.

A PV cell can be modeled as a diode with its characteristic set of I-V curves. Ideally, in reverse bias mode the PV cell would have minimal current flow. Hence, the thermal profile is expected to be homogeneous with a low standard deviation from the mean. However, imperfections (shunts) in the cell give rise to sites of decreased resistances across the cell junction, causing considerable current to flow through it, and resulting in generation of 
heat (hotspots). The graphs in Figure 14 clearly show the change in temperature across the various imperfections (shunts) in the cell as highlighted in the ROIs from Figure 10(c). The length of the different ROI lines corresponds to a set number of pixels of the line and is plotted against temperature. ROI 4 is a region that acts as a control temperature for the PV cell in a non-defected area. Qualitatively, the standard deviation of ROI 4 in contrast to the other ROIs is less in magnitude, suggesting the presence of localized shunts.

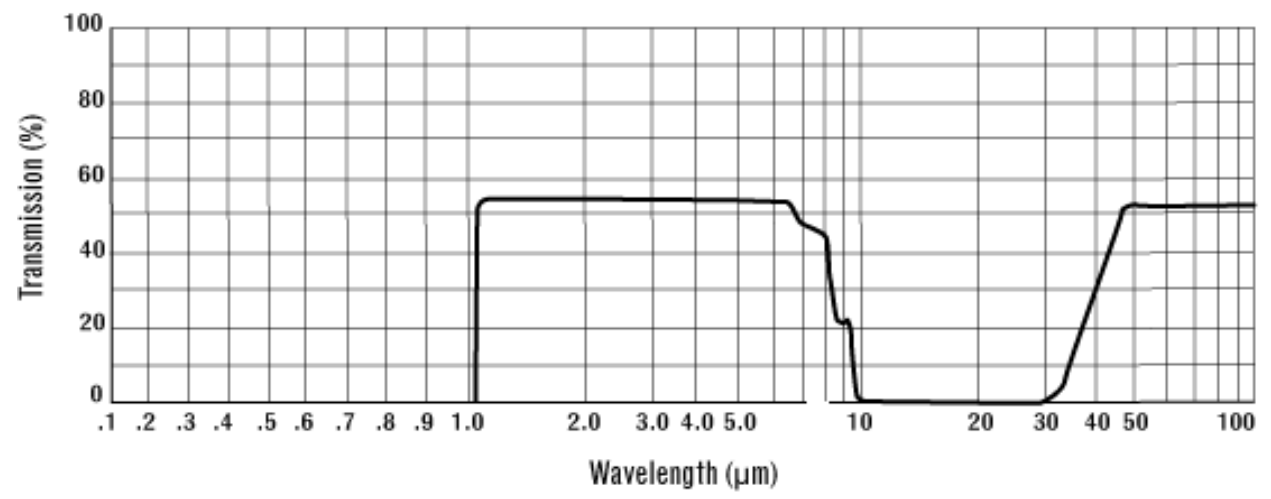

Figure 12. Dependence of wavelength on transmission of light in silicon

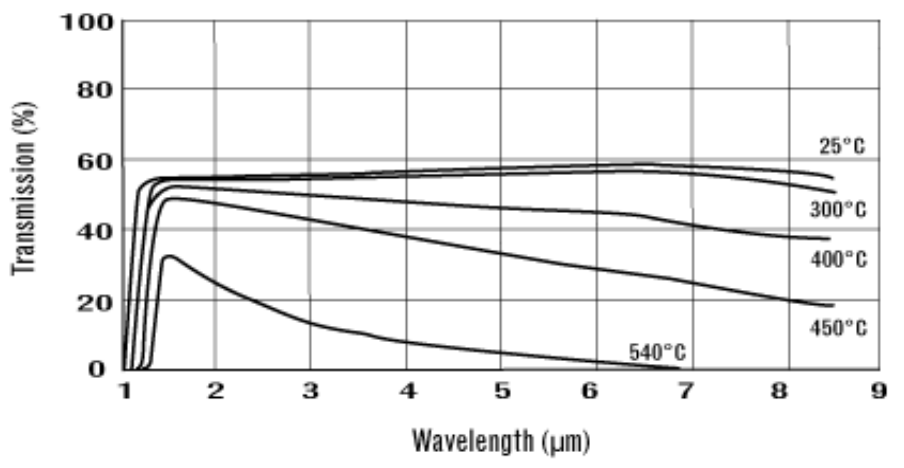

Figure 13. Effect of temperature and wavelength on transmission of light in silicon

Ohmic (linear) shunts often occur underneath the front side metallization or at the cell edges. Performing a measurement at a forward bias and a second measurement under the same reverse bias allows distinguishing between linear shunts and other loss mechanisms, because only Ohmic shunts result in a similar signal under forward and reverse bias. The emitter of silicon solar cells has a thickness in the order of only $0.3 \mathrm{~mm}$. A scratch at the surface of a solar cell across the emitter layer brings the p-n junction to the surface with a high density of recombination centers. Thus, scratches act like nonlinear edge shunts. However, if the indentation of the scratch is fairly deep it may act as a crack resulting in an emitter layer to be established across the scratch. This layer shorts the emitter against the base contact; hence, an Ohmic (linear) shunt appears along the scratch. As can be observed, the scratch represents a dominant hot spot in both the forward and reverse biased images confirming it as an Ohmic shunt. Defects can also be found through Electro-Luminescence in reverse bias. In contrast to the image of forward bias mode of Figure 9(a), Figure 10(a) also shows areas of weak shunts towards the cell edges. These shunts may be regions of zener breakdown causing quantum mechanical tunneling of carriers through the band gap, which is the dominant breakdown mechanism for highly doped p-n junctions. However, the quantitative thermal measurement of local I-V characteristics of these shunts is possible only by lock-in thermography. Because, EL was conducted using a DC power supply and weaker shunts were identified in the reverse bias mode of operation, performing EL using a DC source may prove to be an interesting alternative to lock-in thermography for a fraction of the cost. 

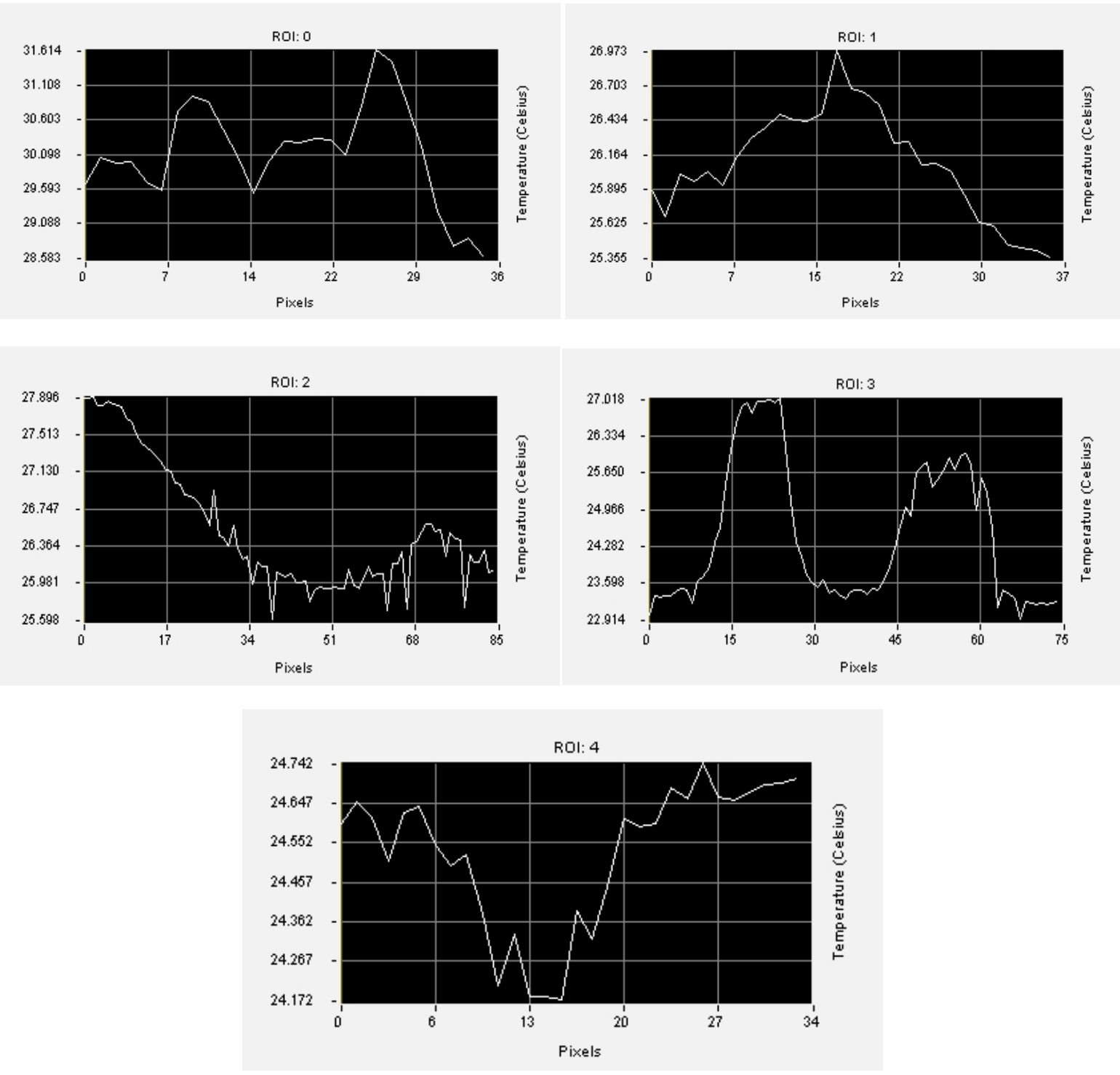

Figure 14. Temperature variations as a function of the length of ROI (given in pixels)

To judge the performance of the two proposed fusion routines namely; PCA and Wavelet along with their different implementations (i.e. fixed weighted average method, adaptive weighted fusion method, comparison method, PCA fusion method, and wavelet fusion methodaverage comparison), a set of the most widely usedfusion quality criteria can be applied to resulted images. These metrics include: (a) mean and standard variance, which evaluates the strength of the signal intensity probability distribution. (b) Signal to Noise Ratio SNR; defines the level of noise by computing the ratio of the fused image mean value to its standard deviation. Also the Peak Signal-to-Noise Ratio or PSNR is often used as a quality measurement between the original and a compressed image. The higher the PSNR, the better the quality of the compressed or reconstructed image is. Here this metric is used to measure the quality difference between the fused image and the raw images, as in Equation (5);

$$
P S N R=10 \log \frac{L^{2}}{R M S E^{2}}
$$

Where, $\mathrm{L}$ is the gray levels of the image and RMSE is the Root-Mean-Square Error RMSE, which is a frequently used measure of the differences between values predicted by a model or an estimator and the values actually observed from the process being modeled or estimated. It can also be used to measure the difference of the fused 
image and the raw images. Other criteria include, Spatial Frequency, and the Distort Extent, where higher distortion means lower quality. All the above mentioned criteria are tabulated in Table 1; also, Figure 15 shows a one-level wavelet fusion of the images from the three channels.

Table 1. The evaluation results of the fusion results

\begin{tabular}{ccccccccccc}
\hline & Mean & STD & SNR & Entropy & SF & CE & DE & RMSE & MI & PSNR \\
\hline Visual & 163.47 & 75.817 & 2.1561 & 6.4762 & 36.3959 & & & & & \\
MWIR & 82.8168 & 90.359 & 0.9165 & 6.484 & 9.3292 & & & & & \\
LWIR & 92.2778 & 75.602 & 1.2206 & 7.0694 & 11.9324 & & & & & \\
PCA Average & 112.855 & 39.761 & 2.8383 & 7.1566 & 12.9707 & $-1.10 \mathrm{E}-05$ & 68.733 & 76.9141 & -3.675 & 10.6887 \\
AWA & 26.7243 & 34.209 & 0.7812 & 5.7588 & 17.7487 & 0.0016 & 86.1307 & 114.3454 & -2.258 & 7.3735 \\
MAX & 204.123 & 43.593 & 4.6824 & 6.425 & 17.8345 & $-1.10 \mathrm{E}-05$ & 91.2677 & 122.8383 & 1.3323 & 6.8095 \\
MIN & 35.5998 & 43.888 & 0.8111 & 6.1287 & 27.4603 & $2.19 \mathrm{E}-05$ & 77.2552 & 109.58 & 1.4943 & 7.7501 \\
Wavelet & 81.0398 & 103.86 & 0.7803 & 3.6387 & 23.2035 & 1.201 & 88.1467 & 102.294 & -5.11 & 9.4422 \\
\hline
\end{tabular}

Where CE represents Cross Entropy, DE is Distort Extent and MI is Mutual Information

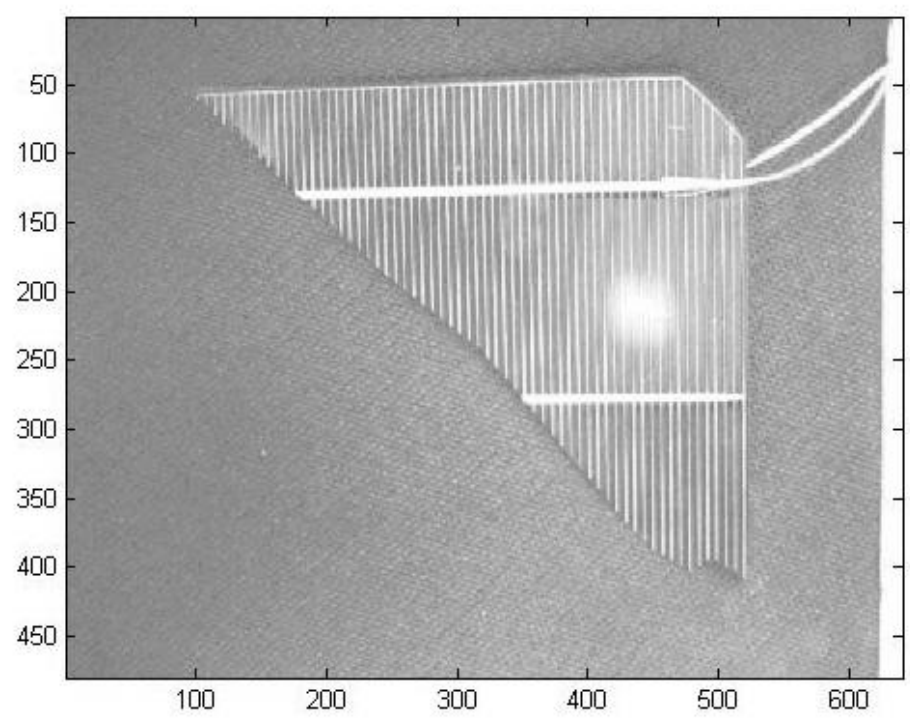

Figure 15. One-Level Wavelet fusion

\section{Conclusions}

This manuscript presented a new method to evaluate and classify PV cell shunts and scratches in real time, using a fused imagery approach. The information from the three imaging sources, MWIR, LWIR and CCD are combined to benefit the PV cell assessment in two folds; first it exposes the extent of facial scratches by monitoring its thermal signature; second not only it detects shunts but also classifies them into Ohmic or Non-Ohmic shunt by fusing the MWIR forward and reverse bias acquisitions.

The proposed fused routines, PCA and Wavelet allowed for a real-time fusion code to accommodate productions environment. The modified SVD-PCA fusion proved to be an efficient approach; however the current fusion assessment metrics might provide conflicting judgment. 
Future work will focus on analyzing and developing a suitable fusion assessment metric for PV cell inspection.

\section{References}

Acciani, G., Falcone, O., \& Vergura, S. (2010). Typical defects of PV-cells. Industrial Electronics (ISIE), 2010 IEEE International Symposium on July 4-7, 2745-2749. http://dx.doi.org/10.1109/ISIE.2010.5636901

Augarten, Y., Abbott, M. D., Trupke, T., Bardos, R., Hartmann, H. P., Gupta, R., ... Breitenstein, O. (2007). Detection and Isolation of Localized Shunts in Industrial Silicon Solar Cells Using PL Imaging. 22nd European Photovoltaic Solar Energy Conference, September 3-7, Milan, Italy.

Bauer, J., Breitenstein, O., \& Wagner, J. M. (2009). Lock-in Thermography: A Versatile Tool for Failure Analysis of Solar Cells. ASM International, 3, 6-12.

Breitenstein, O., Rakotoniaina. J. P., Al Rifai, M. H., \& Werner, M. (2004). Shunt Types in Crystalline Silicon Solar Cells. Progress in Photovoltaics: Research and Applications, 12(7), 529-538. http://dx.doi.org/10.1002/pip.544.

Colvin, J. (2009). Comparative Failure Analysis of Photovoltaic Devices. Conference Proceedings from the 35th International Symposium for Testing and Failure Analysis, San Jose, California, November 15-19, USA, 149-156.

Fuyuki, T., Kondo, H., Yamazaki, T., Takahashi, Y., \& Uraoka, Y. (2005). Photographic Surveying of Minority Carrier Diffusion Length in Polycrystalline Silicon Solar Cells by Electroluminescence. Applied Physics Letters, 86, 262108. http://dx.doi.org/10.1063/1.1978979

Galland, A. (2012). Clean \& Green: Best Practices in Photovoltaic's Report. Retrieved from http://www.clca.columbia.edu/Clean\&Green-Photovoltaics.pdf

Hill, P. R., Bull, D. R., \& Canagarajah, C. N. (2005). Image fusion using a new framework for complex wavelet transforms. Image Processing, 2005. ICIP 2005. IEEE International Conference on September 11-14, vol. 2, II-1338-41. http://dx.doi.org/10.1109/ICIP.2005.1530311

Hong, G., \& Zhang, Y. (2003). High Resolution Image Fusion Based on Wavelet and HIS Transformations. 2nd GRSS/ISPRS Joint Workshop on Remote Sensing and Data Fusion over Urban Areas, May 22-23, Piscataway, NJ, USA, 99-104. http://dx.doi.org/10.1109/DFUA.2003.1219966

Hui, L., Manjunath, B. S., \& Mitra, S. K. (1994). Multi-sensor image fusion using the wavelet transform. Image Processing, 1994. Proceedings. ICIP-94., IEEE International Conference, November 13-16, vol. 1, 51-55. http://dx.doi.org/10.1109/ICIP.1994.413273

Kasemann, M., Grote, D., Walter, B., Kwapil, W., Trupke, T., Augarten, Y., ... \& Warta, W. (2008). Luminescence Imaging for the Detection of Shunts on Silicon Solar Cells. Progress in Photovoltaics: Research and Applications, 16(4), 297-305. http://dx.doi.org/10.1002/pip.812

Lanir, J., Maltz, M., Yatskaer, I., \& Rotman, S. R. (2006). Comparing Multispectral Image Fusion Methods for a Target Detection Task. Information Fusion, 2006 9th International Conference on July 10-13, pp. 1-5. http://dx.doi.org/10.1109/ICIF.2006.301787

Malchow, D. (2009). NIR Trends: Photoluminescence Inspection of Photovoltaics with SWIR Imaging. In: Photonics Online Newsletter $\quad$ (November http://www.sensorsinc.com/downloads/article_NIRTrends-Photoluminescence-Inspection-of-Photovoltaicswith-SWIR-Imaging-Nov09.pdf

National Renewable Energy Laboratory (NREL) (2012). Retrieved August 15, 2012, from http://www.nrel.gov/solar

Pajares, G., \& de la Cruz, J. M. (2004). A Wavelet-based Image Fusion Tutorial. Pattern Recognition, 37(9), 1855-1872. http://dx.doi.org/10.1016/j.patcog.2004.03.010

Parvataneni, R. (2009). Principal Component Thermography for Steady Perturbation Scenarios. Master Degree Thesis, Clemson University.

Rajic, N. (2002a). Principal Component Thermography. DSTO Aeronautical and Maritime Research Laboratory. Retrieved from http://www.dtic.mil/cgi-bin/GetTRDoc?AD=ADA405857

Rajic, N. (2002b). Principal Component Thermography for Flaw Contrast Enhancement and Flaw depth Characterisation in Composite Structures. Composite Structures, 58(4), 521-528. http://dx.doi.org/10.1016/S0263-8223(02)00161-7 
Ranjan, S., Balaji, S., Panella, R. A., \& Ydstie, B. E. (2011). Silicon solar cell production. Computers and Chemical Engineering, 35, 1439-1453. http://dx.doi.org/10.1016/j.compchemeng.2011.04.017

Saga, T. (2010). Advances in crystalline silicon solar cell technology for industrial mass production. NPG Asia Materials, 2, 96-102. http://dx.doi.org/10.1038/asiamat.2010.82

Schmidt, J., Pohl, P., Bothe, K., \& Brendel, R. (2007). Advances in Contactless Silicon Defect and Impurity Diagnostics Based on Lifetime Spectroscopy and Infrared Imaging. Journal of Applied Physics, 101(7), 073701. http://dx.doi.org/10.1155/2007/92842

Sinha, P., Balas, B., Ostrovsky, Y., \& Russell, R. (2006). Face Recognition by Humans: Nineteen Results All Computer Vision Researchers Should Know About. Proceedings of the IEEE, 94(11), 1948-1962. http://dx.doi.org/10.1109/JPROC.2006.884093

Stay, K. (2007). Getting the Most Out of PV Cells. Photovoltaics, 29(2), 15-16.

St-Laurent, L., Genest, M., Simon, C., \& Maldague, X. (2004). Micro-cracks detection in Photo-Voltaic Cells by Infrared Thermography. Proceeding at 7th International Conference of Quantitative Infrared Thermography (QIRT), July 5-8.

Tachibana, T., Sameshima, T., Kojima, T., Arafune, Koichi Kakimoto, K., et al. (2012). Evaluation of defects generation in crystalline silicon ingot grown by cast technique with seed crystal for solar cells. Journal of Applied Physics, 111(7), 074505-074505-5. http://dx.doi.org/10.1063/1.3700250

Trupke, T., Bardos, R. A., Abbott, M. D., Würfel, P., Pink, E., Augarten, Y., ... Fisher, K. (2007). Progress with Luminescence Imaging for the Characterization of Silicon Wafers and Solar Cells.Proceedings of the 22nd European Photovoltaic Solar Energy Conference, Milan, Italy, pp. 21-31. Retrieved from http://btimaging.com/Pdfs/O1\%20Milan\%20Plenary\%20Overview\%201AP.1.5.pdf

Trupke, T., Bardos, R. A., Schubert, M. C., \& Warta, W. (2006). Photoluminescence Imaging of Silicon Wafers. Applied Physics Letters, 89(4), 044107. http://dx.doi.org/10.1063/1.2234747

Würfel, P., Trupke, T., Puzzer, T., Schäffer, E., Warta, W., \& Glunz, S. W. (2009). Diffusion Lengths of Silicon Solar Cells from Luminescence Images. J. Appl. Phys., 101, 123110. http://dx.doi.org/10.1063/1.2749201

Zhou, Y. (2010). Principal Component Analysis based Image Fusion Routine with Application to Stamping Split Detection. Doctor Degree Thesis, Clemson University.

Zhou, Y., \& Omar, M. A. (2009). Routines for fusing infrared, visible acquisitions, applied to night vision $\begin{array}{lllll}\text { systems. International Journal of } & \text { Optomechatronics, } & 3(1), & 41 .\end{array}$ http://dx.doi.org/10.1080/15599610902717835

Zhou, Y., Mayyas A., Qattawi, A., \& Omar, M. A. (2010). Feature-level and Pixel-level fusion routines when coupled to infrared night-vision tracking scheme. Journal of Infrared Physics and Technology, 53(1), 43-49. http://dx.doi.org/10.1016/j.infrared.2009.08.011 